\title{
High Diagnostic Value of a New Real-Time Pneumocystis PCR from Bronchoalveolar Lavage in a Real-Life Clinical Setting
}

\author{
Markus Unnewehr ${ }^{a}$ Hendrik Friederichs $^{c}$ Patricia Bartsch ${ }^{b}$ Bernhard Schaaf ${ }^{a}$ \\ a Pneumologie, Infektiologie, Intensivmedizin, Medizinische Klinik Nord, Klinikum Dortmund gGmbH, and \\ ${ }^{b}$ Laboratory ÜBAG MVZ Dr. Eberhard und Partner, Dortmund, and ${ }^{\mathrm{C}}$ Institute for Education and Student Affairs, \\ Westfälische Wilhelms-Universität Münster, Münster, Germany
}

\section{Key Words}

Pneumonia · Pneumocystis · Real-time PCR · Sensitivity ·

Specificity

\begin{abstract}
Background: To diagnose Pneumocystis jirovecii pneumonia $(P C P), P C R$ testing in bronchoalveolar lavage (BAL) fluid has recently become an alternative to immunofluorescence testing (IFT); however, its diagnostic accuracy is less clear. Objective: To analyze the diagnostic value of a new semiquantitative real-time PCR (RT-PCR) in BAL in a real-life clinical setting. Methods: Retrospective analysis of all RT-PCR results [semiquantitative: negative, weakly positive, and strongly positive; measured in cycle thresholds (Ct)] in BAL in the period between 2010 and 2014. The diagnosis of PCP was defined by clinical, radiological, and laboratory signs and by treatment initiation. Any positive PCR was compared with subsequent IFT. Results: Of 128 patient samples, 32 had PCP. There is a relevant correlation of high significance between positive PCR Ct and IFT ( $r=-0.7781$, $p<0.001)$, which amounts to about $60 \%$ of the variance. Sensitivity, specificity, and positive predictive values (PPV) of any positive RTPCR were 100, 80, and 63\%, respectively. No patient with negative RT-PCR had PCP. Specificity and PPV are $100 \%$ in strongly positive RT-PCR, whereas they decrease to 80 and
\end{abstract}

$21 \%$ in weakly positive RT-PCR. Conclusion: A negative RTPCR $(C t>45)$ rules out PCP. A strongly positive PCR $(C t<31.5)$ confirms PCP. In these cases, the diagnostic value of the new method is at least equal to the IFT. A weakly positive PCR probably represents pneumocystis colonization and can occur under PCP treatment.

(c) 2016 S. Karger AG, Base

\section{Introduction}

Pneumocystis jirovecii pneumonia (PCP) is the most common opportunistic infection and AIDS-defining disease in HIV patients [1]. The infection also occurs in other forms of immunosuppression, especially in iatrogenic immunosuppression [2]. Nowadays, the causative human pathogenic pneumocystis species is known as $P$. jirovecii (PJ), in the honor of the Czech Otto Jirovec [3].

$\mathrm{PJ}$ is a facultative pathogen. Clinically, PCP must be differentiated from a colonization of patients without clinical signs and symptoms of a PCP. For example, colonization with pneumocystis is shown in nearly half of the COPD patients who are treated with glucocorticoids [4].

M.U. and H.F. contributed equally to this work.

\section{KARGER}

E-Mail karger@karger.com

www.karger.com/res
C 2016 S. Karger AG, Basel

0025-7931/16/0923-0144\$39.50/0
Dr. Markus Unnewehr

Pneumologie, Infektiologie, Intensivmedizin, Medizinische Klinik Nord Münsterstrasse 240

DE-44145 Dortmund (Germany)

E-Mail markus.unnewehr@klinikumdo.de 
High diagnostic accuracy is therefore needed to avoid unnecessary therapy.

Frequently, it can be difficult to diagnose PCP because clinical signs and radiological results are often mild [5]. In order to complete the diagnosis, bronchoalveolar lavage (BAL) gained by bronchoscopy is recommended [6]. Induced sputum can be used alternatively [7]. The microbiological diagnosis is normally based on the microscopic detection of pneumocystis by immunofluorescence testing (IFT) or by other staining methods from BAL or sputum. A culture of pneumocystis is not possible.

The IFT is time-consuming, requires specialized laboratory staff, and is not offered by all laboratories. For some years, a faster and simpler to perform PCR-based diagnosis has been available in clinical routine.

Various groups were able to demonstrate that pneumocystis PCR shows a high sensitivity, so that a negative PCR almost rules out PCP $[8,9]$. On the other hand, high rates of false-positive results do not allow qualitative PCR alone to distinguish between colonization and infection. In contrast to qualitative PCR, semiquantitative real-time PCR (RT-PCR) might be able to differentiate between colonization and infection. However, the exact characteristics are not clear $[10,11]$.

Most laboratories perform their own, individually standardized pneumocystis PCR. This is why the comparability of the various PCR methods is difficult and limited. We have recently developed a RT-PCR, which we use on a regular basis.

This study is supposed to clarify the diagnostic accuracy of our new RT-PCR in the clinical daily routine. In particular, the study analyzes to what extent the new method is suitable to differentiate between PCP and pneumocystis colonization. The study evaluates RT-PCR cutoff values for the detection or exclusion of a PCP. In addition, RT-PCR values were compared with the IFT values.

\section{Methods}

\section{Patients}

All patients from our tertiary care hospital in Dortmund, Germany, on which PCP diagnostics from BAL samples were performed in the period between 2010 and April 2014, were retrospectively included into the study.

The study obtained ethical approval from the responsible Institutional Advisory Board, which is the Ethics Committee of the Chamber of Physicians (Ärztekammer Westfalen-Lippe) and the Medical School of the Westfälische Wilhelms-Universität in Münster, Germany.
Table 1. Ct value and corresponding PCR results

\begin{tabular}{ll}
\hline Ct value & PCR result \\
\hline No Ct & negative \\
$<45$ & positive \\
$31.5-45$ & weakly positive \\
$<31.5$ & strongly positive \\
\hline
\end{tabular}

\section{Definition of PCP}

PCP was diagnosed if all of the following clinical, radiological, and microbiological criteria were met: suitable case history, symptoms and clinical findings, immunosuppressive risk factors (amongst others: HIV, hematological diseases, drug-induced immunosuppression), typical interstitial pulmonary infiltrates in chest X-ray or computed tomography (CT), detection of a positive IFT from BAL or a strongly positive PCR result [5, 12]. Furthermore, a usual PCP therapy, e.g. with high-dosed co-trimoxazole, was required to establish the diagnosis.

\section{Collection of Samples via BAL}

After sedation (fentanyl with midazolam and/or disoprivan) and a local anesthesia with $2 \%$ lignocaine, a standardized BAL was carried out. A total of $100-140 \mathrm{ml}$ of $0.9 \%$ sodium chloride solution was instilled in aliquots of $20 \mathrm{ml}$, aspirated, and immediately sent to the laboratory for the analysis.

\section{Pneumocystis RT-PCR}

The PCR is based on a new method, which we developed during the past 10 years without a commercial purpose (Laboratory ÜBAG MVZ Dr. Eberhard und Partner, Dortmund, Germany). This is why our method is not available on the market. Local laboratory authorities and regulatory bodies approved the method after the usual safety and reliability tests had been passed successfully.

PJ DNA from $400 \mu$ l BAL was purified using the QIAamp DNA Mini Kit ${ }^{\circledR}$ (QIAGEN, Hilden, Germany). A 267-bp fragment from the conserved region of the large subunit of the mitochondrial rRNA was amplified by RT-PCR [13]. Suitable hybridization probes were designed using the LightCycler ${ }^{\circledR}$ Probe Design Software Version 1.0 (Roche Diagnostics).

The results of the RT-PCR were evaluated according to cycle thresholds (Ct). This value is the amount of cycles in the RT-PCR, at which the specific fluorescence signal exceeds the background signal for the first time. Therefore, high Ct values correspond to a low concentration of PJ DNA, low Ct values to a high concentration of PJ DNA (table 1).

The Ct were determined during the development of the test from pooled data from all laboratory investigations in the last 10 years and were defined as the technically closest and best limitations. The threshold of a Ct value of 31.5 was chosen according to the test performance experienced on a daily laboratory work basis when developing the test. It was closest to a minimum false-positive rate (maximum specificity), whereas the threshold of a Ct value of $>45$ seemed to yield a maximum sensitivity.

All samples with a positive result in the RT-PCR were additionally examined microscopically using pneumocystis IFT (see below). Negative PCR results were not examined by IFT, since it was 
Table 2. Patient characteristics and BAL results

\begin{tabular}{|c|c|c|c|}
\hline & \multicolumn{3}{|c|}{ PCP-PCR from BAL $(\mathrm{n}=128)$} \\
\hline & \multirow[t]{2}{*}{ negative $(\mathrm{n}=76)$} & \multicolumn{2}{|l|}{ positive $(\mathrm{n}=52)$} \\
\hline & & $\begin{array}{l}\text { weakly positive } \\
(\mathrm{n}=24)\end{array}$ & $\begin{array}{l}\text { strongly positive } \\
(\mathrm{n}=28)\end{array}$ \\
\hline Mean age, years & 51 & 53 & 48 \\
\hline Range & $16-82$ & $27-82$ & $31-74$ \\
\hline SD & 17 & 17 & 11 \\
\hline Males & $59.2 \%$ & $58.3 \%$ & $82.1 \%$ \\
\hline \multicolumn{4}{|l|}{ HIV-positive } \\
\hline patients, $\mathrm{n}$ & $21(27.6 \%)$ & $9(37.5 \%)$ & $21(75 \%)$ \\
\hline Positive IFT, $\mathrm{n}$ & not examined & 0 & $23(82.1 \%)$ \\
\hline PCP diagnosis, $\mathrm{n}$ & 0 & $5(20.8 \%)$ & $28(100 \%)$ \\
\hline
\end{tabular}

known from internal laboratory tests during the development of the PCR method that a negative pneumocystis PCR result had never shown a positive IFT result.

\section{Pneumocystis IFT}

For the visual detection of pneumocystis cysts in the BAL samples, the AXIS SHIELD Detect IF Pneumocystis carinii ${ }^{\circledR}$ test was used according to manufacturer information (Axis-Shield Diagnostics Ltd., Luna Place, The Technology Park, Dundee, UK).

According to the manufacturer, the test results are to be interpreted as follows: no cysts - negative; one to five cysts - undefined; six or more cysts - positive.

\section{Statistical Evaluation}

Descriptive means and standard deviations were calculated for participants' age, and frequencies were computed for gender and test results. For the group differences, cross-tables were generated and Pearson's $\chi^{2}$ test was performed. Spearman's rank correlation coefficient was used for the calculation of a correlation between PCP-PCR and PCP-IFT. The significance level was set at $\mathrm{p} \leq 0.05$. Sensitivity, specificity, and predictive values were calculated for test characteristics.

In a separate analysis of HIV-positive patients, the receiver operating characteristic (ROC) curve was plotted to illustrate findings of an optimum threshold for HIV-positives, and an area under the curve (AUC) was computed.

\section{Results}

\section{Patients}

A diagnostic workup for PCP from BAL was performed on 128 patients (table 2 ). The mean age of the patients was 51 ( $\mathrm{SD} \pm 17$ ) years. $59.2 \%$ of the patients were male, and $27.6 \%$ were HIV positive. Six patients died, all from PCP (12\%). Thirty-three patients (26\%) were clinically diagnosed with PCP according to the criteria mentioned above.

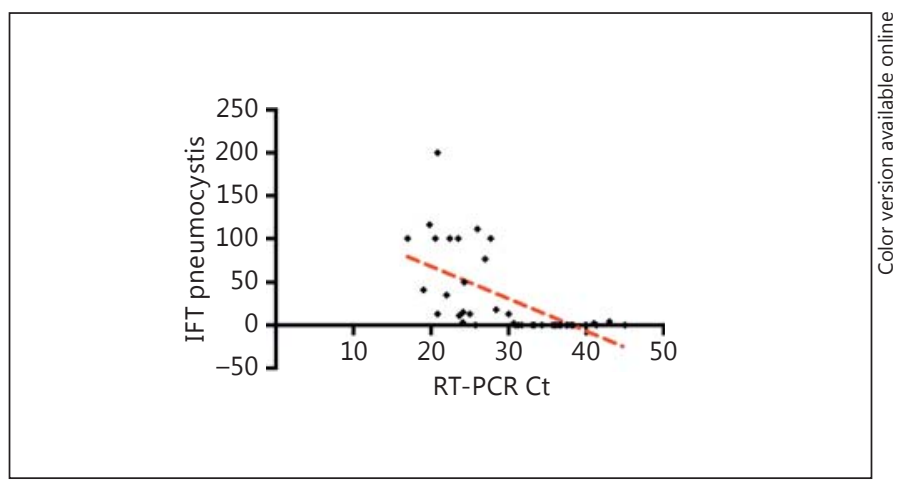

Fig. 1. Correlation of IFT pneumocystis and RT-PCR Ct. There is a Spearman's rank correlation coefficient of high significance (49 pairs; $\mathrm{r}=-0.7781, \mathrm{p}<0.001$ by Pearson's $\chi^{2}$ test).

\section{Pneumocystis RT-PCR in BAL}

Negative RT-PCR: 76 patients (59\%) had a negative PCR (no Ct value). None of these patients had a clinically diagnosed PCP.

Positive RT-PCR: 52 patients $(41 \%)$ had a positive PCP-PCR (Ct $<31.5)$ of which 24 samples were weakly positive and 28 samples were strongly positive.

- Weakly positive RT-PCR (Ct 31.5-45, n = 24): none of these patients had a positive IFT (0\%). Five patients (20.8\%) clinically had PCP.

- Strongly positive RT-PCR (Ct <31.5): 23 of these patients had a positive IFT ( $82.1 \%)$; the IFT was negative or undefined in 5 samples. All 28 patients (100\%) clinically had PCP.

\section{Correlation RT-PCR with IFT}

In Spearman's calculation, there was a negative correlation between Ct in RT-PCR and cysts per visual field in the IFT (49 pairs; $r=-0.7781, p<0.001$ ) (fig. 1 ). Since a low Ct corresponds to a high concentration of pneumocystis DNA, the result of the RT-PCR probably represents the amount of pneumocystis in the BAL.

Patients with strongly positive PCR had a positive IFT significantly more often than patients with weakly positive PCR $\left(\chi^{2}, p<0.0001\right)$.

\section{Test Quality Criteria}

Table 3 shows the statistical characteristics of positive RT-PCR. In the separate analysis of HIV-positive patients, the ROC curve yields an AUC of 0.982 and again an optimum threshold Ct value of 31.5 (fig. 2). 
Table 3. Statistical characteristics of positive RT-PCR

\begin{tabular}{lcccc}
\hline & Sensitivity $(95 \% \mathrm{CI})$ & Specificity $(95 \% \mathrm{CI})$ & PPV (95\% CI) & NPV (95\% CI) \\
\hline RT-PCR & & & & \\
$\quad$ Positive & $100 \%(89-100)$ & $80 \%(71-88)$ & $63 \%(49-76)$ & $100 \%(95-100)$ \\
$\quad$ Weakly positive & $100 \%(48-100)$ & $80 \%(71-88)$ & $21 \%(7-41)$ & $100 \%(95-100)$ \\
$\quad$ Strongly positive & $100 \%(88-100)$ & $100 \%(95-100)$ & $100 \%(88-100)$ & $100 \%(95-100)$ \\
IFT & $70 \%(51-84)$ & $95 \%(88-98)$ & $82 \%(63-94)$ & $82 \%(63-94)$ \\
\hline
\end{tabular}

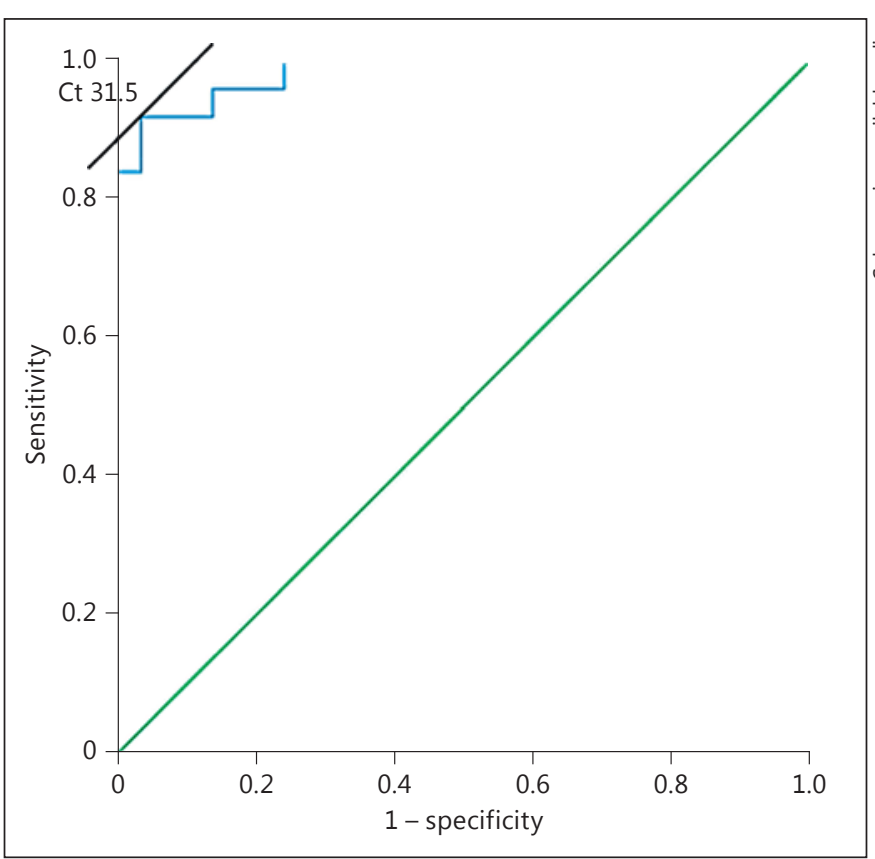

Fig. 2. ROC curve of HIV-positive patients with an AUC of 0.982 and an optimum threshold Ct value of 31.5 (parallel black line).

\section{Discussion}

Nowadays, pneumocystis PCR, especially RT-PCR, is commonly used in diagnosing PCP [14]. A good sensitivity and a strongly negative predictive value (NPV) were confirmed in several studies $[8,15]$. The laboratories normally use specifically developed methods. Interlaboratory standards do not exist on a regular basis.

The investigation performed with our newly developed RT-PCR confirms previous studies which showed that no patient with a negative pneumocystis PCR in BAL had PCP.

A New Pneumocystis PCR from

Bronchoalveolar Lavage
A positive qualitative pneumocystis PCR result does not differentiate between pneumocystis infection and colonization, since the method is highly sensitive and detects pneumocystis DNA regardless of the clinical picture $[16,17]$. However, this differentiation is the most relevant clinically question. Overdiagnosis should be avoided, since PCP therapy can harm the patient [18]. The literature on the positive predictive value (PPV) of the RT-PCR is not uniform $[11,15]$. Especially the cutoff values of the quantitative PCR techniques used have not been tested for the usefulness in daily routine. In addition, many different PCR methods are in use $[9,19-21]$.

In the present study, the cutoff value for a positive test result was defined as a $\mathrm{Ct}$ value of 45 . A subdivision in 'weakly positive' and 'strongly positive' occurred at a Ct value smaller than 31.5. Comparisons with other studies are difficult due to the variety of performed PCR tests. Fillaux et al. [9], for example, found a sensitivity of $100 \%$ at a Ct value of 28 and a specificity of $100 \%$ at a Ct value of $<22$. The variety of measurement units also does not allow a comparison. Chumpitazi et al. [22] determined a cutoff of 54.3 major surface glycoprotein copies per milliliter, Fujisawa et al. [10] used 30 copies per tube, and Mühlethaler et al. [14] defined a positive result at a PCR value of $>1.450$ pathogens $\cdot \mathrm{ml}^{-1}$.

The evaluation of the 'weakly positive' results remains difficult, as our study and other studies show. A total of $20.8 \%$ of our patients with a 'weakly positive' RT-PCR actually had a clinically proven PCP. This means a falsepositive test result for PCP in $79.2 \%$ of the cases, corresponding to the positive predictive value (PPV) of mere $21 \%$. None of the 'weakly positive' PCRs was confirmed by IFT. Methodically, this might be explained partly by starting the PCP therapy before performing the bronchoscopy. Since initiation of a PCP therapy in severe cases must not be delayed by waiting for the BAL results, some patients were treated a few days before diagnostic 
bronchoscopy. As shown by others, PJ DNA might be detected by PCR, while the IFT is negative under therapy [17].

Our study shows for the first time that a cutoff of a Ct value of $>45$ and $<31.5$ is a reliable marker in detecting and ruling out PCP when using our RT-PCR method. Within these cutoff values, RT-PCR was at least equivalent to IFT.

Our results, the comparison with the literature, and the development across the years show that RT-PCR is a promising method $[11,23]$. It has recently been stressed by the Open Science Collaboration that innovation points out paths that are possible; replication points out paths that are likely' [24]. Therefore, we think that our results will further pave the way for this promising method.

In this context, it is remarkable that in countries with limited health care resources, PCR from sputum nowadays seems to be of higher diagnostic value and more cost-effective than staining methods from BAL [7].

The conditions of our study represent the clinical daily routine in a large infectious diseases department in a tertiary care hospital in an urban area in Germany. In order to not change these 'real-life' conditions, we intentionally did not limit the inclusion of patients. The transfer of our results to other patient groups has to take these circumstances into account.

In our study, negative PCR results were not controlled by IFT, since it was known from previous laboratory tests that a negative pneumocystis PCR result has never shown a positive IFT result. This could limit the value of negative PCR. We do not believe that hereby the general significance of our study, especially concerning positive PCR, will be severely biased.

The variable to evaluate was the PCR. Since this was measured during daily clinical routine, it was part of the PCP diagnosis components. This common problem possibly limits the significance of our results due to incorporation bias. However, in a systematic review by Whiting et al. [25], the authors could not detect a significant change in the relative diagnostic odds ratio when the index test was incorporated as a part of the reference standard.

During the study period between 2010 and 2014, the value of the PCR was not clear and still subject to scientific investigation. The detection of pneumocystis has been further improved with the PCR [26] but is still not a perfect parameter to solely rely on to establish the diagnosis. Therefore, the diagnosis of PCP should still be based on a combination of clinical symptoms and signs, radiological imaging, and microbiology, especially if the PCR result is not negative or strongly positive [20].

\section{Summary and Clinical Conclusion}

The semiquantitative RT-PCR practically rules out PCP if it is negative (Ct $>45$, NPV 100\%) and confirms the diagnosis if it is strongly positive $(\mathrm{Ct}<31.5$, PPV $100 \%)$. In these cases, the diagnostic value of the new method is at least equal to the IFT. A weakly positive RTPCR probably represents colonization with pneumocystis and can be found in patients under PCP treatment. A negative IFT does not rule out PCP completely (NPV $82 \%)$.

\section{Statement of Ethics}

The study obtained ethical approval from the Ethics Committee of the Chamber of Physicians (Ärztekammer Westfalen-Lippe) and the Medical School of the Westfälische Wilhelms-Universität in Münster, Germany.

\section{Financial Disclosure and Conflicts of Interest}

The authors declare that they have no conflicts of interest.

\section{References}

Mussini C, Manzardo C, Johnson M, et al: Patients presenting with AIDS in the HAART era: a collaborative cohort analysis. AIDS Lond Engl 2008;22:2461-2469.

- Blum T, Roth A, Mauch H, Erbes R, Lode H: Pneumocystis jiroveci pneumonia in immunocompromised patients without AIDS - a case series (in German). Dtsch Med Wochenschr 2006;131:1515-1520.
Stringer JR, Beard CB, Miller RF, Wakefield AE: A new name for pneumocystis from humans and new perspectives on the hostpathogen relationship. Emerg Infect Dis 2002; 8:891-896.

4 Maskell NA, Waine DJ, Lindley A, et al: Asymptomatic carriage of Pneumocystis jiroveci in subjects undergoing bronchoscopy: a prospective study. Thorax 2003;58:594-597.
Roux A, Gonzalez F, Roux M, et al: Update on pulmonary Pneumocystis jirovecii infection in non-HIV patients. Médecine Mal Infect 2014; 44:185-198.

6 Dalhoff K, Ewig S, Höffken G, et al: Recommendations for the diagnosis, therapy and prevention of pneumonia in the immunocompromised host (in German). Pneumol Stuttg Ger 2002;56:807-831. 
7 Harris JR, Marston BJ, Sangrujee N, DuPlessis D, Park B: Cost-effectiveness analysis of diagnostic options for pneumocystis pneumonia (PCP). PLoS One 2011;6:e23158.

8 Alanio A, Desoubeaux G, Sarfati C, et al: Realtime PCR assay-based strategy for differentiation between active Pneumocystis jirovecii pneumonia and colonization in immunocompromised patients. Clin Microbiol Infect Off Publ Eur Soc Clin Microbiol Infect Dis 2011;17:1531-1537.

9 Fillaux J, Malvy S, Alvarez M, et al: Accuracy of a routine real-time PCR assay for the diagnosis of Pneumocystis jirovecii pneumonia. J Microbiol Methods 2008;75:258-261.

$\rightarrow 10$ Fujisawa T, Suda T, Matsuda H, et al: Realtime PCR is more specific than conventional PCR for induced sputum diagnosis of Pneumocystis pneumonia in immunocompromised patients without HIV infection. Respirol Carlton Vic 2009;14:203-209.

11 Huggett JF, Taylor MS, Kocjan G, et al: Development and evaluation of a real-time PCR assay for detection of Pneumocystis jirovecii DNA in bronchoalveolar lavage fluid of HIVinfected patients. Thorax 2008;63:154-159.

12 Kanne JP, Yandow DR, Meyer CA: Pneumocystis jiroveci pneumonia: high-resolution CT findings in patients with and without HIV infection. AJR Am J Roentgenol 2012; 198:W555-W561.

13 Wakefield AE: DNA sequences identical to Pneumocystis carinii f. sp. carinii and Pneumocystis carinii f. sp. hominis in samples of air spora. J Clin Microbiol 1996;34:1754-1759.
14 Mühlethaler K, Bögli-Stuber K, Wasmer S, et al: Quantitative PCR to diagnose Pneumocystis pneumonia in immunocompromised nonHIV patients. Eur Respir J 2012;39:971-978.

15 Flori P, Bellete B, Durand F, et al: Comparison between real-time PCR, conventional PCR and different staining techniques for diagnosing Pneumocystis jiroveci pneumonia from bronchoalveolar lavage specimens. J Med Microbiol 2004;53:603-607.

16 Reid AB, Chen SC-A, Worth LJ: Pneumocystis jirovecii pneumonia in non-HIV-infected patients: new risks and diagnostic tools. Curr Opin Infect Dis 2011;24:534-544.

17 Nevez G, Raccurt C, Jounieaux V, Dei-Cas E, Mazars E: Pneumocystosis versus pulmonary Pneumocystis carinii colonization in HIVnegative and HIV-positive patients. AIDS Lond Engl 1999;13:535-536.

18 Hardak E, Neuberger A, Yigla M, et al: Outcome of Pneumocystis jirovecii pneumonia diagnosed by polymerase chain reaction in patients without human immunodeficiency virus infection. Respirol Carlton Vic 2012;17: 681-686.

19 Fillaux J, Berry A: Real-time PCR assay for the diagnosis of Pneumocystis jirovecii pneumonia. Methods Mol Biol Clifton NJ 2013;943: 159-170.
20 Robert-Gangneux F, Belaz S, Revest M, et al: Diagnosis of Pneumocystis jirovecii pneumonia in immunocompromised patients by realtime PCR: a 4-year prospective study. J Clin Microbiol 2014;52:3370-3376.

21 Tasaka S, Tokuda H: Recent advances in the diagnosis of Pneumocystis jirovecii pneumonia in HIV-infected adults. Expert Opin Med Diagn 2013;7:85-97.

22 Chumpitazi BFF, Flori P, Kern J-B, et al: Characteristics and clinical relevance of the quantitative touch-down major surface glycoprotein polymerase chain reaction in the diagnosis of Pneumocystis pneumonia. Med Mycol 2011;49:704-713.

23 Robberts FJL, Liebowitz LD, Chalkley LJ: Polymerase chain reaction detection of Pneumocystis jiroveci: evaluation of 9 assays. Diagn Microbiol Infect Dis 2007;58:385-392.

24 Open Science Collaboration: Psychology. Estimating the reproducibility of psychological science. Science 2015;349:aac4716.

-25 Whiting P, Rutjes AWS, Reitsma JB, Glas AS, Bossuyt PMM, Kleijnen J: Sources of variation and bias in studies of diagnostic accuracy: a systematic review. Ann Intern Med 2004; 140:189-202

26 Wilson JW, Limper AH, Grys TE, Karre T, Wengenack NL, Binnicker MJ: Pneumocystis jirovecii testing by real-time polymerase chain reaction and direct examination among immunocompetent and immunosuppressed patient groups and correlation to disease specificity. Diagn Microbiol Infect Dis 2011;69: 145-152.
A New Pneumocystis PCR from

Bronchoalveolar Lavage 